Pacific Journal of Mathematics

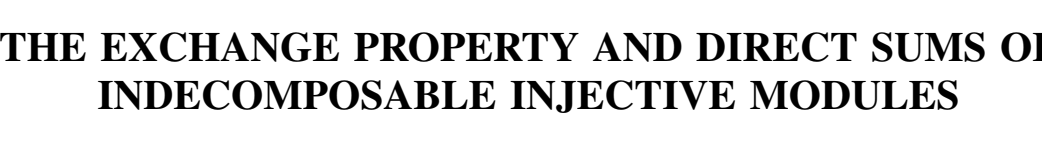




\title{
THE EXCHANGE PROPERTY AND DIRECT SUMS OF INDECOMPOSABLE INJECTIVE MODULES
}

\author{
KUNIO Yamagata
}

This paper contains two main results. The first gives a necessary and sufficient condition for a direct sum of indecomposable injective modules to have the exchange property. It is seen that the class of these modules satisfying the condition is a new one of modules having the exchange property. The second gives a necessary and sufficient condition on a ring for all direct sums of indecomposable injective modules to have the exchange property.

Throughout this paper $R$ will be an associative ring with identity and all modules will be right $R$-modules.

A module $M$ has the exchange property [5] if for any module $A$ and any two direct sum decompositions

$$
A=M^{\prime} \oplus N=\sum_{i \in I} \oplus A_{i}
$$

with $M^{\prime} \cong M$, there exist submodules $A_{i}^{\prime} \subseteq A_{i}$ such that

$$
A=M^{\prime} \oplus \sum_{i \in I} \oplus A_{i}^{\prime} \text {. }
$$

The module $M$ has the finite exchange property if this holds whenever the index set $I$ is finite. As examples of modules which have the exchange property, we know quasi-injective modules and modules whose endomorphism rings are local (see [16], [7], [15] and for the other ones [5]).

It is well known that a finite direct $\operatorname{sum} M=\bigoplus_{i=1}^{n} M_{i}$ has the exchange property if and only if each of the modules $M_{i}$ has the same property ([5, Lemma 3.10]). In general, however, an infinite direct sum $M=\bigoplus_{i \in I} M_{i}$ has not the exchange property even if each of $M_{i}$ 's has the same property. On the other hand, Fuller [8] has recently proved that every module over a generalized uniserial ring has the exchange property (c.f., see [9, Theorem 9 and corollary to Lemma 12]).

Therefore, two interesting questions arise:

(1) When does the infinite direct sum $M=\bigoplus_{i \in I} M_{i}$ of modules $M_{i}(i \in I)$ with the exchange property have the same property?

(2) What ring $R$ has the property that every module $M$ has the exchange property?

In this paper we consider these two problems for the class of modules $M$ which are direct sums of indecomposable injectives and 
completely make answers to them for such a class of modules. In $\S 1$ we show a sufficient condition for a direct sum of modules with local endomorphism rings to have the finite exchange property. In $\S 2$ we prove the following results $\left(1^{\prime}\right)$ and $\left(2^{\prime}\right)$.

( $\left.1^{\prime}\right)$ A module $M$ which is a direct sum of indecomposable injective modules has the exchange property if and only if it has the finite exchange property, and moreover any of these assertions is equivalent to that the Jacobson radical of the endomorphism ring $\operatorname{End}_{R}(M)$ of $M$ is $\left\{f \in \operatorname{End}_{R}(M) \mid \operatorname{Ker} f\right.$ is essential in $\left.M\right\}$.

( $\left.2^{\prime}\right)$ A ring $R$ satisfies the ascending chain condition for (meet-) irreducible right ideals if and only if every direct sum of indecomposable injective modules has the exchange property.

It is not known whether the exchange and finite exchange properties coincide, so the first equivalence in $\left(1^{\prime}\right)$ is meaningful. Since any direct summand of a module with the exchange property has also the same property as mentioned above, the second equivalence in $\left(1^{\prime}\right)$ trivially includes [2, Corollaire 5] concerning a problem on an indecomposable decomposition of a direct summand of the module which is a direct sum of indecomposable injectives (this is a problem of Matlis). is a strengthening of [19, Theorem 1] and, as seen in it, such a ring in $\left(2^{\prime}\right)$ has interesting properties concerning the Krull-Remak-SchmidtAzumaya's theorem and a problem of Matlis. If a module $M$ is quasi-injective, all properties in $\left(1^{\prime}\right)$ are also valid for $M$, but conversely neither of them implies the quasi-injectivity of $M$. In $\S 3$ we show this fact with an example which means that the class of all modules with the exchange property which are direct sums of indecomposable injectives is a new one of modules with the same property. In $\S 4$ we generalize the results of Chamard [3, Théorème 3] and Yamagata [17, Theorem 4] which are obtained from the point of view of a problem of Matlis.

The author wishes to express hearty thanks to Prof. Tachikawa for his advices.

1. A semi-T-nilpotent system. We will recall some definitions and elementary results from [9] and [10]. A family $\left\{M_{i}\right\}_{i \in I}$, with an infinite index set $I$, which consists of modules $M_{i}$ whose endomorphism rings are local is called (resp. semi-) T-nilpotent system if for any family of nonisomorphisms $\left\{f_{i_{n}}: M_{i_{n}} \rightarrow M_{i_{n+1}} \mid n \geqq 1\right\}$ (resp. $i_{n} \neq i_{n^{\prime}}$ for $\left.n \neq n^{\prime}\right)$ and any element $x_{i_{1}} \in M_{i_{1}}$, there is an integer $m$ depending on $x_{i_{1}}$ such that $f_{i_{m}} f_{i_{m-1}} \cdots f_{i_{1}}\left(x_{i_{1}}\right)=0$. If $\mathscr{A}$ is the full subcategory of the category of all right modules whose objects are isomorphic to direct sums of $M_{i}$ 's, then it is said to be the induced category from $\left\{M_{i}\right\}_{i \in I}$ and we denote by $\mathscr{J}$ the class of all morphisms $f$ in $\mathscr{A}$ such that for two objects $X=\bigoplus_{j \in J} X_{j}$ and $Y=\bigoplus_{k \in K} Y_{k}$ of $\mathscr{A}$ with $f: X \rightarrow$ 
$Y$ and indecomposable modules $X_{j}$ and $Y_{k}$, each $\pi_{k} f \kappa_{j}$ is a nonisomorphism where $\kappa_{j}$ is the canonical injection of $X_{j}$ to $X$ and $\pi_{k}$ the projection of $Y$ to $Y_{k}$. In [9] we then know the quotient category $\mathscr{A}=\mathscr{A} / \mathscr{J}$ is $C_{3}$-completely reducible abelian.

For a morphism $f: M \rightarrow N$ and a submodule $M_{0}$ of $M, f \mid M_{0}: M_{0} \rightarrow$ $N$ denotes the restriction of $f$ to $M_{0}$. We denote by $\operatorname{End}_{R}(M)$ an endomorphism ring of a right module $M_{R}$ over a ring $R$.

Now we write the proposition, without proof, which will play an important role in our proofs.

Proposition 1.1 ([12], [13]). Let $\left\{M_{i}\right\}_{\imath \in I}$ be an infinite family of modules with local endomorphism rings and $M=\bigoplus_{\imath \in I} M_{\imath}$. Then the following conditions are equivalent.

(i) $\left\{M_{i}\right\}_{i \in I}$ is a semi-T-nilpotent system.

(ii) $\mathcal{J} \cap \operatorname{End}_{R}(M)$ is the Jacobson radical of $\operatorname{End}_{R}(M)$.

In this case, each direct summand of $M$ is also a direct sum of indecomposable modules which are isomorphic to some $M_{i}$.

Lemma 1.2. For two modules $M_{1}$ and $M_{2}$, let

$$
M=M_{1} \oplus M_{2}
$$

and $\rho$ a projection of $M$ to $M_{1}$. Then for a nonzero submodule $N$ of $M$ with $N \cap M_{2}=0$ the restriction $\rho \mid N$ is a monomorphism. If, further, $\rho(N)$ is a direct summand of $M$, then there exists a submodule $N_{1}$ of $M_{1}$ such that $M=N \oplus N_{1} \oplus M_{2}$.

Proof. The first assertion is clear. For the rest let $\rho(N)$ be a direct summand of $M, M=\rho(N) \oplus M^{\prime}$ and $\rho$ a monomorphism on $N$. By the modular law, we then have

$$
M_{1}=\rho(N) \oplus N_{1}
$$

with a projection $\pi$ of $M_{1}$ to $\rho(N)$ where $N_{1}=M_{1} \cap M^{\prime}$. We consider the decomposition

$$
M=\rho(N) \oplus N_{1} \oplus M_{2} .
$$

It is then easy to see that the projection of $M$ to $\rho(N)$ be $\pi \rho$ and the restriction $\pi \rho \mid N$ of $\pi \rho$ to $N$ is an isomorphism by the first part of this lemma. As a consequence, we obtain the desired decomposition

$$
M=N \oplus N_{1} \oplus M_{2} .
$$

The following corollaries are essentially proved in [9] but we include proofs for completeness. In them, without proofs, we will 
use some properties for completely reducible objects in $\mathscr{A}$ but they are easily proved in the same way as for completely reducible modules (see [9, p. 331-332]).

CoRollary 1.3. Let $M$ be a direct sum of indecomposable modules $M_{i}(i \in I)$, where each $M_{i}$ has a local endomorphism ring, and $\left\{N_{j}\right\}_{j \in J}$ an independent set of indecomposable submodules of $M$ with local endomorphism rings such that it is a semi-T-nilpotent system. Then, if $\sum_{j \in F} \oplus N_{j}$ is a direct summand of $M$ for every finite subset $F \subset J$, there exists a subset $K \subset I$ such that

$$
M=\sum_{j \in J} \oplus N_{j} \oplus \sum_{k \in K} \oplus M_{k} .
$$

REMARK. If $J$ is finite, the finite direct sum $\sum_{j \in J} \oplus N_{j}$ has the exchange property by [15, Proposition 1] and [5, Lemma 3.10] and is a direct summand of $M$ by hypothesis. Hence there exists a subset $K \subset I$ such that $M=\sum_{j \in J} \oplus N_{j} \oplus \sum_{k \in K} \oplus M_{k}$.

Proof. We assume $J$ is infinite. Let $\mathscr{A}$ and $\mathscr{J}$ be as above and $\kappa: N=\sum_{j \in J} \oplus N_{j} \rightarrow M$ an inclusion map. For a morphism $f$ in $\mathscr{A}$ we denote by $\bar{f}$ the induced morphism of $f$ in the quotient category $\bar{A}=\mathscr{A} / \mathscr{J}$. Since $N_{j_{1}} \oplus \cdots \oplus N_{j_{n}}$ is a direct summand for any finite subset $\left\{j_{1}, \cdots, j_{n}\right\}$ of $J$ by assumption, the restriction of $\bar{\kappa}$ to $\bar{N}_{j_{1}} \oplus \cdots \oplus \bar{N}_{j_{n}}$ is then an injection in $\overline{\mathscr{A}}_{\text {. This will imply that } \bar{\kappa}}$ is an injection in $\bar{A}$.

To show this we suppose that the kernel $\bar{K}=\operatorname{Ker} \bar{\kappa}$ is not zero. Then there is a finite subset $\left\{j_{1}, \cdots, j_{n}\right\} \subset J$ such that $\bar{K} \cap$ $\left(\bar{N}_{j_{1}} \oplus \cdots \oplus \bar{N}_{j_{n}}\right) \neq 0$, because $\bar{A}$ is a $C_{3}$-abelian category and $\bar{N}=$ $\bigoplus_{j \in J} \bar{N}_{j}$ in $\bar{A}^{n}\left(\left[9\right.\right.$, Theorem 7]). Hence $\bar{\kappa}\left(\bar{K} \cap \sum_{k=1}^{n} \oplus \bar{N}_{j_{k}}\right) \neq 0$ by the fact that $\bar{\kappa} \mid \sum_{k=1}^{n} \oplus \bar{N}_{j_{k}}$ is injective in $\mathscr{A}$, a contradiction.

Then, since the category $\mathscr{A}$ is $C_{3}$-completely reducible abelian, the morphism $\bar{\kappa}: \bar{N} \rightarrow \bar{M}$ splits and by the note just before this corollary there is a subset $K \subset I$ such that

$$
M=\sum_{i \in I-K} \oplus M_{i} \oplus \sum_{k \in K} \oplus M_{k}
$$

and

$$
\begin{aligned}
\bar{M} & =\bar{N} \oplus \sum_{k \in K} \oplus \bar{M}_{k} \\
& =\sum_{i \in I-K} \oplus \bar{M}_{i} \oplus \sum_{k \in K} \oplus \bar{M}_{k} .
\end{aligned}
$$

Let the projection of $M$ to $\sum_{j \in I-K} \oplus M_{i}$ be $\rho$. Then in (3) the projection of $\bar{M}$ to $\sum_{i \in I-K} \bar{M}_{i}$ is clearly $\bar{\rho}$ and so $\bar{\rho} \circ \bar{\kappa}$ is a bijection of $\bar{N}$ 
onto $\sum_{\imath \in I-K} \oplus \bar{M}_{i}$ in view of (2) and (3). This means that there is a morphism $\phi$ of $\sum_{\imath \in I-K} \oplus M_{\imath}$ to $N$ such that $\bar{\phi} \circ \bar{\rho} \circ \bar{\kappa}=1_{\bar{N}}$ and $\bar{\rho} \circ \bar{\kappa} \circ \bar{\phi}=$ $1_{\Sigma_{i \in I-K} \oplus \bar{M}_{i}}$. Hence we obtain that $\phi \circ(\rho \circ \kappa)-1$ and $(\rho \circ \kappa) \circ \phi-1$ belong to $\mathscr{J}\left(\operatorname{End}_{R}(N)\right)=\mathscr{f} \cap \operatorname{End}_{R}(N)$ and $\mathscr{J}\left(\operatorname{End}_{R}\left(\sum_{\imath \in I-K} \oplus M_{\imath}\right)\right)=$ $\mathscr{J} \cap \operatorname{End}_{R}\left(\sum_{i \in I-K} \oplus M_{i}\right)$ respectively. We will show that $\rho \circ \kappa$ is an isomorphism of $N$ to $\sum_{\imath-I-K \in K} \oplus M_{\imath}$.

First, $\dot{\phi} \circ(\rho \circ \kappa)-1 \in \mathscr{J}\left(\operatorname{End}_{R}(N)\right)$ implies that $\dot{\phi} \circ(\rho \circ \kappa)$ is invertible, because $\mathscr{f}\left(\operatorname{End}_{R}(N)\right)$ is the Jacobson radical by Proposition 1.1. The morphism $\rho \circ \kappa$ is hence a monomorphism.

Secondly, to show that $\rho \circ \kappa$ is an epimorphism it suffices to show that the family $\left\{M_{i}\right\}_{i \in I-K}$ is a semi-T-nilpotent system by the same reason in the first part. Now since $\bar{N}=\sum_{j \in J} \oplus \bar{N}_{j}$ is isomorphic to $\sum_{i \in I-K} \oplus \bar{M}_{\imath}$, there is a bijection $\sigma: J \rightarrow I-K$ such that $\bar{N}_{j} \cong \bar{M}_{\sigma(j)}$ for every $j \in J$ because $\mathscr{A}$ is a completely reducible $C_{3}$-abelian category (see the note before this corollary). It is therefore easy to see that $N_{j}$ is isomorphic to $M_{\sigma(\jmath)}$ for every $j \in J$ on account of the facts that $f \cap \operatorname{End}_{R}\left(N_{j}\right)$ and $f \cap \operatorname{End}_{R}\left(M_{\sigma(\jmath)}\right)$ are the Jacobson radicals of $\operatorname{End}_{R}\left(N_{j}\right)$ and $\operatorname{End}_{R}\left(M_{\sigma(j)}\right)$ respectively. Hence the assumption that $\left\{N_{\jmath_{j \in J}}\right.$ is a semi-T-nilpotent system implies that the family $\left\{M_{i}\right\}_{i \subset I-K^{*}}$ is also semi-T-nilpotent, as desired.

Now then, since $(\rho \circ \kappa)(N)=\rho(N)=\sum_{\imath \in I-K} \oplus M_{i}$ is a direct summand of $M$, we can apply Lemma 1.2 to our case and have that

$$
M=N \oplus \sum_{k \in K} \oplus M_{k},
$$

which completes the proof of the corollary.

COROLlary 1.4. Let $M$ be an infinite direct sum of indecomposable modules $M_{i}(i \in I)$ with local endomorphism rings. Assume the family $\left\{M_{2}\right\}_{2 \in I}$ is a semi-T-nilpotent system and let $\left\{N_{n}\right\}_{n \geqq 1}$ be a family of direct summands of $M$ such that $N_{n} \subseteq N_{n+1}$ for all integers $n \geqq 1$. Then the union $\bigcup_{n \geqq 1} N_{n}$ of the family $\left\{N_{n}\right\}_{n \geqq 1}$ is also a direct summand of $M$.

Proof. Since, according to Proposition 1.1, the union $\bigcup_{n \geqq 1} N_{n}$ is also a direct sum of indecomposable modules with local endomorphism rings, it is an immediate consequence of Corollary 1.3.

For two modules $M=\bigoplus_{i \in I} M_{\imath}$ and $N=\bigoplus_{j \in J} N_{j}$ we can represent every homomorphism $f$ of $M$ to $N$ as a column summable matrix $\left(f_{j_{2}}\right)$, that is, for the injections $\kappa_{i}$ of $M_{i}$ to $M$ and projections $\pi_{j}$ of $N$ to $N_{j}(i \in I, j \in J), f_{j_{\imath}}=\pi_{j} f \kappa_{i}: M_{i} \rightarrow N_{j}$ and, for any $x \in M$ and $i \in I$ $f_{j i}\left(\rho_{i}(x)\right)=0$ for almost all $j \in J$ where $\rho_{i}$ is the projection of $M$ onto $M_{\imath}$. Hence, in this case we may denote that $f(x)=\sum_{j} \pi_{j} f(x)=$ $\sum_{i, j} f_{j i}\left(\rho_{i}(x)\right)$ for any $x \in M$ and $f_{i}=\sum_{j \in J} f_{j i}$ (see [9], p. 332). 
A submodule $N$ of $M$ is essential in $M\left(N \subseteq^{\prime} M\right)$ if $N \cap L \neq 0$ for all nonzero submodules $L$ of $M$ and $M$ is uniform if every nonzero submodule is essential in $M$. In the following we will denote the kernel of a morphism $f$ by $\operatorname{Ker} f$.

Lemma 1.5. Let $M$ be a direct sum of uniform modules $M_{2}(i \in I)$ and $f=\left(f_{j i}\right) \in \operatorname{End}_{R}(M)$. Then Ker $f$ is essential in $M$ if and only if each $\operatorname{Ker} f_{j i}$ is essential in $M_{i}$ for all $i, j \in I$.

Proof. Suppose that $\operatorname{Ker} f_{j i}$ is essential in $M_{i}$ for all $i, j \in I$. Then to show that $\operatorname{Ker} f \subseteq^{\prime} M$ it suffices to show that $\operatorname{Ker} f \cap M_{i} \subseteq^{\prime}$ $M_{i}$ for all $i \in I$. Now contrary to it, suppose that for some $i \in I$, $\operatorname{Ker} f \cap M_{i}$ is not essential in $M_{i}$ or equivalently $\operatorname{Ker} f \cap M_{i}=0$ by reason of the uniformity of $M_{\imath}$. Then for $0 \neq x_{\imath} \in M_{i}$ there exists a finite subset $\left\{j_{1}, \cdots, j_{n}\right\} \subseteq I$ such that $0 \neq f_{i}\left(x_{i}\right)=\sum_{k=1}^{n} f_{j_{k} i}\left(x_{i}\right)$ and $f_{j_{2}}\left(x_{i}\right)=0$ for all $j \neq j_{k}$, where $f_{i}=\sum_{j \in J} f_{j i}$. Because the restriction $f_{i}=f: M_{i}: M_{i} \rightarrow M$ is a monomorphism. On the other hand, by hypothesis, $\quad\left(\bigcap_{k=1}^{n} \operatorname{Ker} f_{j_{k} i}\right) \cap x_{i} R \neq 0$ and $f_{2}\left(\left(\bigcap_{k=1}^{n} \operatorname{Ker} f_{j_{k} i}\right) \cap x_{i} R\right)=$ $\left(\sum_{k=1}^{n} f_{j_{k^{2}}}\right)\left(\left(\cap_{k=1}^{n} \operatorname{Ker} f_{j_{k^{2}}}\right) \cap x_{\imath} R\right)=0$, a contradiction. Thus Ker $f \cap M_{i}$ is essential in $M_{2}$ for every $i \in I$.

Conversely, we assume that $\operatorname{Ker} f \subseteq M$. Clearly this implies that $\operatorname{Ker} f \cap M_{\imath} \subseteq M_{2}$ by the uniformity of $M_{\imath}(i \in I)$. On the other hand, since $f_{\imath}\left(x_{2}\right)=\sum_{j \in J} f_{j_{2}}\left(x_{i}\right)$ and $f_{j_{2}}\left(x_{i}\right) \in M_{j}$ for every $x_{i} \in M_{2}$, that $f_{i}\left(x_{2}\right)=0$ implies that $f_{j_{2}}\left(x_{2}\right)=0$ for all $j \in I$. Therefore, $\operatorname{Ker} f_{j i} \neq 0$ for all $i, j \in I$, because $\operatorname{Ker} f_{i}=\operatorname{Ker} f \cap M_{i} \neq 0$. As a consequence, Ker $f_{j i} \subseteq^{\prime} M_{i}$ for $i, j \in I$.

Lemma 1.6 ([9], [10]). Let $\left\{M_{i}\right\}_{i \in I}$ be a family of a semi-Tnilpotent system of modules with local endomorphism rings and $M=\bigoplus_{i \in I} M_{\imath}$. Then $S / J$ is a regular ring in the sense of von Neumann and an idempotent of $S / J$ can be lifted to $S$, where $S$ is an endomorphism ring of $M$ and $J$ its Jacobson radical.

This follows from Proposition 1.1, [9, Theorem 7] and [10, Theorem 3].

Proposition 1.7. Let $\left\{M_{\imath}\right\}_{\imath \in I}$ be a family of a semi-T-nilpotent system of modules with local endomorphism rings. Then $M=$ $\bigoplus_{i \in I} M_{i}$ has the finite exchange property.

Proof. Let $S=\operatorname{End}_{R}(M)$ and $J$ the Jacobson radical of $S$. Then $S / J$ is a regular ring and every idempotent is lifted to $S$ by Lemma 1.6. Hence, for every element $s \in S$ there exists an idempotent $e \in S$ such that $s S+J=e S+J$. This shows that $S$ has the exchange 
property as a $S$-module and so $M_{R}$ has the finite exchange property by [17, Theorems 3 and 4$]$.

2. The exchange property. In this section we prove our main theorems being concerned with modules which are direct sums of indecomposable injectives.

First we will continue to consider a general case of modules with local endomorphism rings instead of indecomposable injectives.

Lemma 2.1. Let $M, N$, and $A_{\imath}(i \in I)$ be submodules of a module A such that

$$
A=\sum_{i \in I} \oplus A_{i}=M \oplus N
$$

and, furthermore, let $M$ be a direct sum of indecomposable submodules $M_{j}(j \in J)$ with local endomorphism rings. If $M \cap \sum_{i \in F} \oplus A_{i} \neq 0$ for some finite subset $F$ of $I$, then there exist elements $i_{0} \in F$ and $j_{0} \in J$ such that

$$
A=M_{j_{0}} \oplus A_{i_{0}}^{\prime} \oplus \sum_{i \in I-\left\{i_{0}\right\}} \oplus A_{i}
$$

for a suitable submodule $A_{i_{0}}^{\prime}$ of $A_{i_{0}}$.

Proof. First we remark that, since each $M_{\jmath}(j \in J)$ has a local endomorphism ring, it has the exchange property by [15, Proposition 1], so that any finite direct sum of $M_{j}$ 's has also the exchange property ([5, Lemma 3.10]).

Now by hypothesis there exists a finite subset $J_{0}$ of $J$ such that $\sum_{j \in J_{0}} \oplus M_{j} \cap \sum_{i \in F} \oplus A_{i} \neq 0$. Hence applying the exchange property of $\sum_{j \in J_{0}} \oplus M_{j}$ to the given decomposition $A=\sum_{\imath \in I} \oplus A_{i}$, we have decompositions such that

$$
\begin{array}{r}
A_{i}=B_{\imath} \oplus C_{i}(i \in I), \\
A=\sum_{i \in J} \oplus B_{\imath} \oplus \sum_{i \in I} \oplus C_{i}
\end{array}
$$

and

$$
=\sum_{j \in J_{0}} \oplus M_{j} \oplus \sum_{i \in I} \oplus C_{i} .
$$

Here there exists at least one element $i_{0}$ of $F$ such that $B_{i_{0}} \neq 0$. For, if the contrary were true, $\sum_{i \in F} \oplus B_{i}=0$ and hence $\sum_{i \in F} \oplus$ $A_{\imath}=\sum_{i \in F} \oplus C_{i}$. So $\sum_{j \in J_{0}} \oplus M_{j} \cap \sum_{i \in F} \oplus C_{\imath}=\sum_{j \in J_{0}} \oplus M_{j} \cap \sum_{i \in F} \oplus$ $A_{i} \neq 0$ by the definition of $J_{0}$, which contradicts the decomposition (2). 
Now it is clear that $M^{\prime}=\sum_{j \in J_{0}} \oplus M_{j}$ is isomorphic to $\sum_{i \in I} \oplus B_{i}$ via the restriction $\pi \mid M^{\prime}$ of $\pi$ to $M^{\prime}$, where $\pi$ is the projection of $A$ onto $\sum_{i \in I} \oplus B_{i}$ in the formula (1). It follows that

$$
\pi\left(M^{\prime}\right)=\sum_{j \in J_{0}} \pi\left(M_{j}\right)=B_{i_{0}} \oplus \sum_{i \in I-\left\{i_{0}\right\}} \oplus B_{i} .
$$

Since each $\pi\left(M_{j}\right)$ for $j \in J_{0}$ is isomorphic to $M_{j}$, it has a local endomorphism ring. We can thus apply the Krull-Remak-Schmidt-Azumaya's theorem [1, Theorem 1] to this module $\pi\left(M^{\prime}\right)$ and the projection $\xi$ of $\pi\left(M^{\prime}\right)$ onto $B_{i_{0}}$ in the formula (3). As a consequence, there exists an element $j_{0} \in J_{0}$ such that the restriction $\xi \mid \pi\left(M_{j_{0}}\right)$ is a monomorphism and $\xi \pi\left(M_{j_{0}}\right)$ is a direct summand of $\pi\left(M^{\prime}\right)$ and hence of $B_{i_{0}}$. On the other hand, a simple computation shows that the projection of $A$ to $B_{i_{0}}$ in the decomposition (1) is $\xi \pi$. Thus from these facts and Lemma 1.2 there is a submodule $D_{i_{0}}$ of $B_{i_{0}}$ such that

$$
A=M_{j_{0}} \oplus D_{i_{0}} \oplus \sum_{i \in I-\left\{i_{0}\right\}} \oplus B_{i} \oplus \sum_{i \in I} \oplus C_{i}
$$

because the restriction $\xi \pi \mid M_{j_{0}}$ is clearly a monomorphism. Setting $A_{i_{0}}^{\prime}=C_{i_{0}} \oplus D_{i_{0}}$, we finally have a desired decomposition

$$
A=M_{j_{0}} \oplus A_{i_{0}}^{\prime} \oplus \sum_{i \in I-\left\{i_{0}\right\}} \oplus A_{i} .
$$

From now on we will consider indecomposable injectives.

LeMma 2.2. Every indecomposable injective module is uniform and has a local endomorphism ring.

This is well known (c.f., see [6, §5 Proposition 8]).

Assume $M_{1}$ and $M_{2}$ are indecomposable injectives and $f$ a morphism of $M_{1}$ to $M_{2}$. If $f$ is a nonmonomorphism, then its kernel Ker $f$ is essential in $M_{1}$ by Lemma 2.2 and the converse is, of course, true. This shows that $f$ is a nonisomorphism if and only if $\operatorname{Ker} f$ is essential in $M_{1}$. Under this observation we have

Proposition 2.3. Let $\left\{M_{i}\right\}_{i \in I}$ be an infinite family of indecomposable injective modules and $M=\bigoplus_{i \in I} M_{i}$. Let $S$ be an endomorphism ring of $M_{R}$ and $J$ the Jacobson radical of $S$. Then $J=$ $\left\{f \in S \mid \operatorname{Ker} f \cong \subseteq^{\prime} M\right.$ if and only if the family $\left\{M_{i}\right\}_{i \in I}$ is a semi-Tnilpotent system.

Proof. We will represent every endomorphism $f$ of $M$ as a column summable matrix: $f=\left(f_{j i}\right)$, where $f_{j i}=\pi_{j} f \kappa_{i}$ for the projections $\pi_{j}$ of $M$ onto $M_{j}$ and injections $\kappa_{i}$ of $M_{i}$ into $M(i, j \in I)$. Then, 
in accordance with our earlier notations (see $\S 1$ ), by the above remark we have

$$
\mathscr{J} \cap S=\left\{f=\left(f_{j_{2}}\right) \in S \mid \operatorname{Ker} f_{j i} \subseteq^{\prime} M_{i}\right\}
$$

and by Lemma 1.5

$$
\left\{f=\left(f_{j i}\right) \in S \mid \operatorname{Ker} f_{j i} \subseteq^{\prime} M_{i}\right\}=\left\{f \in S \mid \operatorname{Ker} f \subseteq \subseteq^{\prime} M\right\} .
$$

On the other hand, we know by Proposition 1.1 that the family $\left\{M_{i}\right\}_{i \in I}$ is a semi-T-nilpotent system if and only if $J=\mathscr{J} \cap S$. It follows from them that $\left\{M_{i}\right\}_{i \in I}$ is a semi-T-nilpotent system if and only if $J=\left\{f \in S \mid \operatorname{Ker} f \subseteq^{\prime} M\right\}$, which proves the proposition.

We need more lemmas for the main theorems.

Lemma 2.4. A module $M$ has the exchange property if for any modules $A_{i}(i \in I)$ which are isomorphic to submodules of $M$ and any decomposition $A=\bigoplus_{\imath \in I} A_{\imath}=M^{\prime} \oplus N$ where $M^{\prime} \cong M$, there exist submodules $A_{i}^{\prime} \subseteq A_{\imath}$ such that $A=M^{\prime} \oplus \sum_{i \in I} \oplus A_{i}^{\prime}$.

This is well known in [5, Theorem 8.2] and its proof will be omitted.

Lemma 2.5. Let $G=M \oplus N$ for submodules $M$ and $N$ of a given module $G$. We moreover assume $M=\sum_{i \in I} \oplus M_{i}$, where $\left\{M_{i}\right\}_{i \in I}$ is an infinite family of indecomposable injective submodules of $G$ and a semi-T-nilpotent system. Then if a module $A$ is isomorphic to a submodule of $M$ and contains an injective submodule, there exists a maximal submodule $A_{0}$ of $A$ with the property that $A_{0}$ is a direct sum of indecomposable injective submodules. In this case such a module $A_{0}$ is a direct summand of $A$.

Proof. Let the monomorphism of $A$ to $M$ be $f$ and $E$ an injective submodule of $A$. Then by [1, Theorem 1] and Lemma 2.2, $f(E)$ contains an indecomposable injective submodule isomorphic to some $M_{\imath}$ in view of that $f(E)$ is a direct summand of $M$. This implies that $A$ contains a submodule isomorphic to some $M_{i}$. Now then we can take a family $\left\{A_{n}\right\}_{n \geqq 1}$ of submodules of $A$ such that each $A_{n}$ is a direct sum of indecomposable injectives and $A_{n} \subseteq A_{n+1}$ for any $n \geqq 1$. Then, by Zorn's lemma, we will be done if we can show that the union $A_{0}=\mathrm{U}_{n} A_{n}$ is also a direct sum of indecomposable injectives and, furthermore, a direct summand of $A$.

Since $f$ is a monomorphism, the image $f\left(A_{n}\right)$ of $A_{n}$ by $f$ is also a direct sum of indecomposable injectives and hence $f\left(A_{n}\right)$ is a direct 
summand of $M$ for any $j \in J$ by Corollary 1.3 and Lemma 2.2. Thus the union $\mathrm{U}_{n} f\left(A_{n}\right)$ is also a direct summand of $M$ and a direct sum of indecomposable injectives by Corollary 1.4. Taking account of $f\left(A_{0}\right)=\mathrm{U}_{n} f\left(A_{n}\right)$, we have $M=f\left(A_{0}\right) \oplus N$ for a submodule $N$ of $M$ and $A_{0}$ is a direct sum of indecomposable injectives since $A_{0} \cong f\left(A_{0}\right)$. By the modular law, $f(A)=f\left(A_{0}\right) \oplus f(A) \cap N$. We therefore have $A=A_{0} \oplus f^{-1}(f(A) \cap N)$, where $f^{-1}(f(A) \cap N)$ is the inverse image of $f(A) \cap N$ by $f$, which proves the lemma.

It is clear that the exchange property implies the finite exchange property, but it is not known whether the converse is true in general. However, in our case that modules are direct sums of indecomposable injectives we can conclude this question affirmatively.

THeOREM 2.6. Let $M$ be a module which is a direct sum of indecomposable injective modules and let $S$ be an endomorphism ring of $M_{R}$. Then the following assertions are equivalent.

(i) $M$ has the exchange property.

(ii) $M$ has the finite exchange property.

(iii) The Jacobson radical of $S$ is $\left\{f \in S \mid \operatorname{Ker} f \subseteq^{\prime} M\right\}$.

Proof. Let $M=\sum_{i \in I} \oplus M_{i}$, where every submodule $M_{i}$ is indecomposable injective. If the index set $I$ is finite, then $M$ is clearly injective, so all of the above assertions (i), (ii), and (iii) are true. It therefore suffices to show the theorem for only the case with the infinite index set $I$.

Now let $I$ be an infinite index set. By Proposition 2.3 the assertion (iii) is then equivalent to

(iii') The family $\left\{M_{i}\right\}_{i \in I}$ is a semi-T-nilpotent system.

Thus we will consider (iii') instead of (iii) in the following.

The implication (i) $\Rightarrow$ (ii) is trivial.

(ii) $\Rightarrow$ (iii'). The idea of the proof is due to [9, Lemma 9]. Assume that $M$ has the finite exchange property. Take an arbitrary countable subfamily of $\left\{M_{i}\right\}_{i \in I}$, say $\left\{M_{n}\right\}_{n \geqq 1}$, and nonisomorphisms $f_{n}: M_{n} \rightarrow M_{n+1}(n \geqq 1)$. For every $x \in M_{1}$ we will find an integer $n(x)$ depending on $x$ such that $f_{n(x)} f_{n(x)-1} \cdots f_{1}(x)=0$.

For this put $M_{n}^{\prime}=\left\{x+f_{n}(x) \mid x \in M_{n}\right\}$. It is then clear that $M_{n}^{\prime} \oplus$ $M_{n+1}=M_{n} \oplus M_{n+1}$ for $n \geqq 1$. Since each $M_{i}$ is indecomposable injective, every nonisomorphism $f_{n}$ is only nonmonomorphism, i.e., $\operatorname{Ker} f_{n} \neq 0$. This implies that $M_{n}^{\prime} \cap M_{n} \neq 0$ for every $n \geqq 1$.

It is clear that 
(1)

$$
\begin{aligned}
\sum_{i=1}^{\infty} \oplus M_{i} & =M_{1}^{\prime} \oplus M_{2} \oplus M_{3}^{\prime} \oplus M_{4} \oplus \cdots \oplus M_{2 n-1}^{\prime} \oplus M_{2 n} \oplus \cdots \\
& =M_{1} \oplus M_{2}^{\prime} \oplus M_{3} \oplus M_{4}^{\prime} \oplus \cdots \oplus M_{2 n-1} \oplus M_{2 n}^{\prime} \oplus \cdots
\end{aligned}
$$

and we put

$$
N=M_{1}^{\prime} \oplus M_{3}^{\prime} \oplus \cdots \oplus M_{2 n-1}^{\prime} \oplus \cdots .
$$

Then, applying the fact that $N$ has also the finite exchange property ([5, Lemma 3.10]) to the decomposition (2), we have that $\sum_{i=1}^{\infty} \oplus M_{i}=$ $N \oplus X \oplus Y$ for some submodules $X$ and $Y$ of $\sum_{n=1}^{\infty} \oplus M_{2 n-1}$ and $\sum_{n=1}^{\infty} \oplus M_{2 n}^{\prime}$ respectively. Here, in fact, it will hold $X=0$.

To show this, suppose that $X \neq 0$ contrary. Then by Lemma 2.1 there exists $M_{2 m-1}$ such that

$$
\sum_{i=1}^{\infty} \oplus M_{i}=N \oplus M_{2 m-1} \oplus X^{\prime} \oplus Y
$$

for some submodule $X^{\prime}$ of $X$.

This however contradicts that $0 \neq M_{2 m-1} \cap M_{2 m-1}^{\prime} \subseteq M_{2 m-1} \cap N$. Thus it holds

$$
\sum_{i=1}^{\infty} \oplus M_{i}=N \oplus Y
$$

Now we take an arbitrary nonzero element $x \in M_{1}$ and we let $x=y+z$ with $y \in N$ and $z \in Y$. Considering these $y$ and $z$ in the decompositions $N=\sum_{n=1}^{\infty} \oplus M_{2 n-1}^{\prime}$ and $\sum_{n=1}^{\infty} \oplus M_{2 n}^{\prime}$ respectively, we have

$$
y=\sum_{i=1}^{s}\left(x_{2 i-1}+f_{2 i-1}\left(x_{2 i-1}\right)\right)
$$

and

$$
z=\sum_{i=1}^{s}\left(x_{2 i}+f_{2 i}\left(x_{2 i}\right)\right)
$$

and substituting these expressions for $y$ and $z$, we have

$$
\begin{aligned}
x & =\sum_{i=1}^{s}\left(x_{2 i-1}+f_{2 i-1}\left(x_{2 i-1}\right)\right)+\sum_{i=1}^{s}\left(x_{2 i}+f_{2 \imath}\left(x_{2 i}\right)\right) \\
& =x_{1}+\sum_{i=1}^{2 s-1}\left(f_{\imath}\left(x_{\imath}\right)+x_{i+1}\right)+f_{2 s}\left(x_{2 s}\right) .
\end{aligned}
$$

Therefore, $x=x_{1}, f_{i}\left(x_{i}\right)+x_{\imath+1}=0(1 \leqq i \leqq 2 s-1)$ and $f_{2 s}\left(x_{2 s}\right)=0$, that is, $x_{1}=x, x_{2}=-f_{1}(x), \cdots, x_{2 s}=f_{2 s}\left(x_{2 s-1}\right)$ and $f_{2 s-1}\left(x_{2 s}\right)=0$. By successive substitutions, we obtain $x_{2 s}=(-1)^{2 s-1} f_{2 s-1} \cdots f_{1}(x)$ and, finally, $f_{2 s} f_{2 s-1} \cdots$ $f_{1}(x)=0$. Thus we can put $n(x)=2 s$, which completes the proof of (ii) $\Rightarrow$ (iii'). 
(iii') $\Rightarrow$ (i). We assume the family $\left\{M_{i}\right\}_{i \in I}$ is a semi-T-nilpotent system. Suppose $A=\sum_{j \in J} \oplus A_{j}=M^{\prime} \oplus N$, where $M^{\prime} \cong M$ and each $A_{j}$ is isomorphic to a submodule of $M^{\prime}$. Then, taking account of Lemma 2.4, we will be done if we can find submodules $A_{j}^{\prime}$ of $A_{j}$ $(j \in J)$ such that $A=M^{\prime} \oplus \sum_{j \in J} \oplus A_{j}^{\prime}$.

For this, we will first refine the given decomposition $A=\sum_{j \in J} \oplus$ $A_{j}$. We should note that $M^{\prime}$ is also a direct sum of indecomposable injective submodules $M_{i}^{\prime}(i \in I)$. By Lemma 2.1 there exists at least one element $j_{0} \in J$ such that $A_{j_{0}}$ has a nonzero submodule isomorphic to some $M_{i}^{\prime}$. Let the subset of $J$ of such elements $j_{0} \in J$ be $J_{0}$. By Lemma 2.5 there exist maximal submodules $B_{j}$ of $A_{j}\left(j \in J_{0}\right)$ such that each $B_{j}$ is a direct sum of indecomposable injective submodules of $A_{j}$, in which case every $B_{j}$ is a direct summand of $A_{j}$, say $A_{j}=$ $B_{j} \oplus C_{j}$ for a submodule $C_{j} \subset A_{j}$ for $j \in J_{0}$. Consequently, we have such a refinement of $A=\sum_{j \in J} \oplus A_{j}$ that

$$
A=\sum_{j \in J_{0}} \oplus B_{j} \oplus \sum_{j \in J_{0}} \oplus C_{j} \oplus \sum_{j \in J \rightarrow J_{0}} A_{j},
$$

where $J-J_{0}$ is the complement of $J_{0}$ in $J$ and if $J-J_{0}$ is empty, we put $A_{j}$ in the formula (1) to be zero submodule of $A$ for convenience.

Next we will have that

$$
M^{\prime} \cap\left(\sum_{j \in J_{0}} \oplus C_{j} \oplus \sum_{j \in J-J_{0}} \oplus A_{j}\right)=0 .
$$

For this we suppose that $M^{\prime} \cap\left(\sum_{j \in J_{0}} \oplus C_{j} \oplus \sum_{j \in J-J_{0}} \oplus A_{j}\right) \neq 0$. Then by Lemma 2.1 and the choice of $J_{0}$ there exists $M_{i_{0}}^{\prime}$ such that for a submodule $X_{j_{0}} \subseteq C_{j_{0}}$,

$$
A=M_{i_{0}}^{\prime} \oplus X_{j_{0}} \oplus B_{j_{0}} \oplus \sum_{j \in J-\left\{j_{0}\right\}} \oplus A_{j}
$$

This implies there exists an injective submodule $C_{j_{0}}^{\prime}$ of $C_{j_{0}}$ which is isomorphic to $M_{i_{0}}^{\prime}$. However, in this case we have that $B_{j_{0}} \oplus C_{j_{0}}^{\prime}$ is a direct summand of $A_{j_{0}}$ and a direct sum of indecomposable injective submodules, which contradicts the maximality of $B_{j_{0}}$.

Now we can exchange the complement $N$ of $M^{\prime}$ for a direct sum of submodules of $A_{j}(j \in J)$. For this let the projection of $A$ onto $\sum_{j \in J_{0}} \oplus B_{j}$ in (1) be $\rho$. The family $\left\{M_{i}^{\prime}\right\}_{i \in I}$ is semi-T-nilpotent by hypothesis, and so is $\left\{\rho\left(M_{i}^{\prime}\right)\right\}_{\imath \in I}$ because the restriction $\rho \mid M^{\prime}$ of $\rho$ to $M^{\prime}$ is a monomorphism by (2) and Lemma 1.2. Using Corollary 1.3 the image $\rho\left(M^{\prime}\right)$ therefore is a direct summand of $\sum_{j \in J_{0}} \oplus B_{j}$ and there is a subset $K \subset J_{0}$ such that $\sum_{j \in J_{0}} \oplus B_{j}=\rho\left(M^{\prime}\right) \oplus \sum_{k \in K} \oplus B_{k}$ and, consequently we have $A=\rho\left(M^{\prime}\right) \oplus \sum_{k \in K} \oplus B_{k} \oplus \sum_{j \in J_{0}} \oplus C_{j} \oplus$ 
$\sum_{j \in J-J_{0}} \oplus A_{j}$. Computing the projection of $A$ to $\rho\left(M^{\prime}\right)$ and by Lemma 1.2, we therefore have a decomposition

$$
A=M^{\prime} \oplus \sum_{k \in K} \oplus B_{k} \oplus \sum_{j \in J_{0}} \oplus C_{j} \oplus \sum_{j \in J-J_{0}} \oplus A_{j},
$$

which completes the proof of the implication (iii') $\Rightarrow$ (i). Thus we conclude the theorem.

The original definition of the exchange property given in the introduction is due to Crawly and Jónsson [5]. However, we will consider the following weaker exchange property, too ([10]).

Definition. A direct summand $M$ of a module $A$ has the exchange property in $A$ if for any direct sum decomposition $A=\sum_{i \in I} \oplus$ $A_{i}$, there exist submodules $A_{i}^{\prime} \subseteq A_{i}$ such that $A=M \oplus \sum_{i \in I} A_{i}^{\prime}$.

We recall that for a ring $R$ a right ideal $I$ is (meet-) irreducible provided $I \neq R$ and $I=I_{1} \cap I_{2}$ implies $I=I_{1}$ or $I=I_{2}$ for all right ideals $I_{1}$ and $I_{2}$ or $R$.

THEOREM 2.7. The following conditions are equivalent.

(i) A ring $R$ satisfies the ascending chain condition for irreducible right ideals.

(ii) Any direct sum of indecomposable injective modules has the exchange property.

(iii) Any direct sum of indecomposable injective modules has the finite exchange property.

(iv) Any direct summand of the module $M$ which is a direct sum of indecomposable injective modules has the exchange property in $M$.

(v) For any direct sum $M$ of indecomposable injective modules, the Jacobson radical of the endomorphism ring $\operatorname{End}_{R}(M)$ is $\left\{f \in \operatorname{End}_{R}(M) \mid \operatorname{Ker} f \subseteq M\right\}$.

Proof. The equivalences (ii) $\Leftrightarrow$ (iii) $\Leftrightarrow$ (v) are trivial from Theorem 2.6, and (ii) $\Rightarrow$ (iv) follows from [5, Lemma 3.10]. The implication (iv) $\Rightarrow$ (i) is contained in [19, Theorem 1].

(i) $\Rightarrow$ (ii): Let $M=\sum_{i \in I} \oplus M_{i}$, where $M_{i}$ is indecomposable injective for any $i \in I$. If $I$ is finite, then $M$ is clearly injective, so it has the exchange property ([16, Lemma 2]). If $I$ is infinite, the family $\left\{M_{i}\right\}_{i \in I}$ is a semi-T-nilpotent system by [19, Theorem 1 and Lemma 2]. Therefore, $M$ has the exchange property by Proposition 2.3 and Theorem 2.6 .

3. Example. Here we show the existence of modules which are not quasi-injective but isomorphic to direct sums of indecomposable 
injectives and have the exchange property.

We first note that a quasi-injective module $M$ over a ring $R$ is injective by the criterion of Fuchs [7, Lemma 2] provided that $M$ has the property that some finite direct sum of copies of $M$ contains an element with a zero annihilator right ideal or, equivalently, contains a submodule isomorphic to the ring $R$.

The ring $R$ regarded as a right (left) module over itself will be written $R_{R}\left({ }_{R} R\right)$.

LEMma 3.1. For a ring $R$ the following conditions are equivalent.

(i) $R$ is right perfect and its injective hull $E\left(R_{R}\right)$ is projective, $\Sigma$-(quasi-) injective.

(ii) $R$ is left perfect and its injective hull $E\left({ }_{R} R\right)$ is projective, $\Sigma$-(quasi-) injective.

REMARK. By the above note the " $\Sigma$-quasi-injective" and " $\Sigma$-injective" are coincident in Lemma 3.1 .

Proof. We will only prove that (i) implies (ii) as the converse follows by symmetry.

Assume (i). Since $R$ is right perfect, $E\left(R_{R}\right)$ has an indecomposable direct sum decomposition, $E\left(R_{R}\right)=\sum_{i=1}^{m} \oplus P_{i}$, where each $P_{i}$ is injective projective right module. Let $R=e_{1} R \oplus \cdots \oplus e_{n} R$ for primitive idempotents $e_{i}$. Then there is an integer $\kappa(i)$ such that $P_{i} \cong e_{\kappa(i)} R$ for any $1 \leqq i \leqq m$. Let $\left\{P_{j}\right\}_{j=1}^{s}$ be a subclass of mutually nonisomorphic projective modules of $\left\{P_{i}\right\}_{i=1}^{m}$ such that each $P_{i}(1 \leqq i \leqq m)$ is isomorphic to some $P_{j}(1 \leqq j \leqq s)$ (here, if need, the indecies are renumbered) and we put $M=P_{1} \oplus \cdots \oplus P_{s}$, then a right ideal $I=e_{\kappa(1)} R \oplus \cdots$ $\oplus e_{\kappa(s)} R$ is isomorphic to $M$. Since $M$ is clearly $\Sigma$-injective and faithful, so is then also $I$. Thus, by [4, Theorem 1.3], $E\left({ }_{R} R\right)$ is projective, and $R$ is left perfect and contains faithful, $\Sigma$-injective left ideal $\sum_{i=1}^{l} \oplus E\left(S_{i}\right)$, where $\left\{S_{i}\right\}_{i=1}^{l}$ is the representative class of simple left ideals which are nonisomorphic mutually and $E\left(S_{i}\right)$ an injective hull contained in $R$. As a consequence, $E\left({ }_{R} R\right)$ is $\Sigma$-injective because $E\left({ }_{R} R\right)$ is isomorphic to a submodule of a finite direct sum of copies of $\sum_{i=1}^{l} \oplus E\left(S_{i}\right)$. This completes the proof.

Now then, we suppose $R$ is a (left and right) perfect ring such that $E\left(R_{R}\right)$ is projective and $E\left({ }_{R} R\right)$ is not projective (for the existence of such a ring, see Müller [14] and Colby and Rutter [4]). Then, $E\left(R_{R}\right)=\sum_{i=1}^{m} \oplus P_{i}$, where each $P_{i}$ is indecomposable injective for $1 \leqq$ $i \leqq m$ and, since the radical of every projective right module over a right perfect ring is small, any infinite family of modules each of which is isomorphic to some $P_{i}$ is a $T$-nilpotent system ([12, Theorem 3]). On the other hand, an infinite direct sum $M=\bigoplus_{i \in I} M_{\imath}$ with 
$M_{i} \cong E\left(R_{R}\right)$ is not quasi-injective by Lemma 3.1. Thus $M$ is the desirable module having the exchange property by Proposition 2.3 and Theorem 2.6.

4. Applications. We will generalize the theorems of Chamard [3, Théorème 3] and Yamagata [18, Theorem 4].

We recall definitions. A submodule $N$ of a module $M$ is said to be closed if it has no proper essential extension in $M$, that is, if $N \subseteq{ }^{\prime}$ $X$ for any submodule $X$ of $M$, then $N=X$. A module $M$ is said to be well-complemented in case any finite intersection of closed submodules of $M$ is also closed.

Lemma 4.1. Let $M$ be a direct sum of indecomposable injective modules $M_{i}(i \in I)$ and $N$ a direct summand of $M$. If $N$ is well complemented, then $N$ is also a direct sum of indecomposable injective submodules.

Proof. By [1, Theorem 1] it is clear $N$ has a nonzero indecomposable injective submodule, so we can choose a maximal independent set $\left\{N_{j}\right\}_{j \in J}$ of indecomposable injective submodules of $N$. Put $N_{0}=$ $\sum_{j \in J} \oplus N_{j}$.

We will show $N=N_{0}$. To show this take an arbitrary nonzero element $x \in N$. Then there exists an injective hull $E(x R)$ of $x R$ in $N$ by [18, Lemma 2] and it is a finite direct sum of indecomposable injectives by [1, Theorem 1], say $E(x R)=E_{1} \oplus \cdots \oplus E_{n}$. By the maximality of $\left\{N_{j}\right\}_{j \in J}$, it is evident that $N_{0} \cap E_{i} \neq 0$ for $1 \leqq i \leqq n$. Then, since $N$ is well-complemented by hypothesis, this will imply $E_{i} \subseteq N_{0}$ for $1 \leqq i \leqq n$ and so $x \in E(x R) \leqq N_{0}$, which means $N=N_{0}$.

Because there exists a finite subset $\left\{j_{1}, \cdots, j_{m}\right\} \subseteq J$ such that $\sum_{k=1}^{m} \oplus N_{j_{k}} \cap E_{i} \neq 0$ for $1 \leqq i \leqq n$. Since $\sum_{k=1}^{m} \oplus N_{j_{k}}$ and $E_{i}$ are injective, they are closed in $N$ and so is $\sum_{k=1}^{m} \oplus N_{j_{k}} \cap E_{i}$ by hypothesis of $N$ for any $1 \leqq i \leqq n$. Then, since $E_{i}$ is an essential extension of $\sum_{k=1}^{m} \oplus N_{j_{k}} \cap E_{i}$ by Lemma 2.2, it must be that $E_{i}=\sum_{k=1}^{m} \oplus N_{j_{k}} \cap E_{i}$ and therefore $E_{i} \cong \sum_{k=1}^{m} \oplus N_{j_{k}}$ for any $i$. Consequently $x \in E(x R) \subseteq$ $\sum_{k=1}^{m} \oplus N_{j_{k}} \subseteq N$, which concludes the lemma.

Under the same assumptions as in Lemma 4.1, we remark that $N$ has no proper essential submodule which is a direct sum of indecomposable injectives from the proof of Lemma 4.1. This is first shown by Chamard [3, Lemma 4.1].

Proposition 4.2. Let $M$ be a direct sum of indecomposable injective modules $M_{i}(i \in I)$ and $N$ a direct summand of $M ; M=N \oplus$ $N^{\prime}$. If $N$ is well-complemented, then $N$ has the exchange property 
and $N$ and $N^{\prime}$ are also direct sums of indecomposable injective submodules.

Proof. By Lemma 4.1, $N$ is a direct sum of indecomposable injective submodules $N_{j}(j \in J)$. To show that $N$ has the exchange property we will check the property (iii) in Theorem 2.6.

Let $S$ be an endomorphism ring of $N_{R}$ and $J$ its Jacobson radical. We must show that $J=\left\{f \in S \mid \operatorname{Ker} f \subseteq^{\prime} N\right\}$. The inclusion $J \subseteq$ $\{f \in S \mid \operatorname{Ker} f \subseteq N\}$ is known in [2, p. 564]. Conversely take an arbitrary element $f \in S$ with $\operatorname{Ker} f \subseteq N$. To show that $f \in J$, it is enough to show that $1-f$ is an isomorphism.

First we will prove that $1-f$ is a monomorphism. If $\operatorname{Ker}(1-f) \neq$ $0, \quad x R \cap \operatorname{Ker} f \neq 0$ for any nonzero element $x \in \operatorname{Ker}(1-f)$ since Ker $f \subseteq \subseteq^{\prime} N$. There is hence a nonzero element $y$ of $x R$ with $f(y)=0$ and so $y=(1-f)(y)$ which must imply $y=0$, because $y \in \operatorname{Ker}(1-f)$, a contradiction.

Next we will prove that $1-f$ is an epimorphism. Since $1-f$ is a monomorphism, $(1-f)(N)$ is also a direct sum of indecomposable injectives. Take an arbitrary nonzero element $x \in N$. Then $x R \cap$ $\operatorname{Ker} f \neq 0$, that is, there is a nonzero element $y \in x R \cap \operatorname{Ker} f$. We therefore have $x R \cap(1-f)(N) \neq 0$, because $y=(1-f)(y) \in x R \cap$ $(1-f)(N)$. This shows that $(1-f)(N)$ is essential in $N$, so that $N=(1-f)(N)$. Because $N$ has no proper essential submodule which is a direct sum of indecomposable injectives by the remark just before this proposition.

Thus we have shown that $N$ has the exchange property. We can then exchange $N^{\prime}$ for $\sum_{k \in K} \oplus M_{k}$ for some subset $K \subset I, M=$ $N \oplus \sum_{k \in K} \oplus M_{k}$. This implies that $N^{\prime} \cong \sum_{k \in K} \oplus M_{k}$, which completes the proof of the proposition.

Let $M_{R}$ be any nonsingular module over a ring $R$, that is, $M \neq$ 0 and if $x I=0$ for $x \in M$ and essential right ideal $I$ of $R$, then $x=$ 0 . It is then well known that the lattice of all closed submodules of $M$ is complete and so $M$ is clearly well-complemented (c.f., see [6, Corollary 8, p. 61]). Thus we can sharpen [18, Theorem 4] and [11, Proposition 4].

Corollary 4.3. Let $M, N$, and $N^{\prime}$ be as above. If $N$ is nonsingular, then it has the exchange property and so $N$ and $N^{\prime}$ are also direct sums of indecomposable injective submodules.

\section{REFERENCES}

1. G. Azumaya, Corrections and supplementaries to my paper concerning Krull- 
Remak-Schmidt's theorem, Nagoya Math. J., 1 (1950), 117-124.

2. A. Cailleau and G. Renault, Anneau associé à une somme directe infinite de modules quasi-injectifs, Arch. der Math., 21 (1970), 561-566.

3. J. Y. Chamard, Modules riches en co-irréductible et sous-modules compléments, C.

R. Acad. Sc. Paris, t. 264 (1967), 987-990.

4. R. R. Colby and E. A. Rutter, Jr., Generalizations of QF-3 algebras, Trans. Amer. Math. Soc., 153 (1971), 371-386.

5. P. Crawly and B. Jónsson, Refinements for infinite direct decompositions of algebraic systems, Pacific J. Math., 14 (1964), 797-855.

6. C. Faith, Lectures on Injective Modules and Quotient Rings, Lecture Notes in Mathematics, 49, Springer-Verlag, 1967.

7. L. Fuchs, On quasi-injective modules, Annali della Scuola Norm. Sup. Pisa, 23 (1969), 541-546.

8. K. R. Fuller, On generalized uniserial rings and decompositions that complement direct summands, Math. Ann., 200 (1973), 175-178.

9. M. Harada and Y. Sai, On categories of indecomposable modules I, Osaka J. Math., 7 (1970), 323-344.

10. M. Harada, On categories of indecomposable modules II, ibid., 8 (1971), 309-321.

11. - Note on categories of indecomposable modules, (to appear).

12. M. Harada and H. Kanbara, On categories of projective modules, Osaka J. Math., 8 (1971), 471-483.

13. H. Kanbara, Note on Krull-Remak-Schmidt-Azumaya's theorem, ibid., 9 (1972), 409-413.

14. B. J. Müller, Dominant dimension of semi-primary rings, J. Reine Angew Math., 232 (1968), 173-179.

15. R. B. Warfield, Jr., A Krull-Schmidt theorem for infinite sums of modules, Proc. Amer. Math. Soc., 22 (1969), 460-465.

16. - Decompositions of injective modules, Pacific J. Math., 31 (1969), 263-276.

17. - Exchange rings and decompositions of modules, Math. Ann., 199 (1972), $31-36$.

18. K. Yamagata, Nonsingular rings and Matlis' problem, Sci. Rep. Tokyo Kyoiku Daigaku, Sect. A, 11 (1972), 114-121.

19. - A note on a problem of Matlis, Proc. Japan Acad., 49 (1973), 145-147.

Received July 17, 1973 and in revised form April 9, 1974.

TOKYO UNIVERSITY OF EDUCATION

OTSUKA, BUNKYO-KU, TOKYo, JAPAN 



\section{PACIFIC JOURNAL OF MATHEMATICS}

EDITORS

RICHARD ARENS (Managing Editor)

University of California

Los Angeles, Calıfornia 90024

R. A. Beaumont

University of Washington

Seattle, Washington 98105
J. DugundJI

Department of Mathematics

University of Southern California

Los Angeles, California 90007

D. Gilbarg and J. Milgram

Stanford University

Stanford, California 94305

\section{ASSOCIATE EDITORS}
E. F. BECKENBACH
B. H. NeumanN
F. WOLF
K. YOSHIDA

\section{SUPPORTING INSTITUTIONS}

UNIVERSITY OF BRITISH COLUMBIA

CALIFORNIA INSTITUTE OF TECHNOLOGY

UNIVERSITY OF CALIFORNIA

MONTANA STATE UNIVERSITY

UNIVERSITY OF NEVADA

NEW MEXICO STATE UNIVERSITY

OREGON STATE UNIVERSITY

UNIVERSITY OF OREGON

OSAKA UNIVERSITY
UNIVERSITY OF SOUTHERN CALIFORNIA

STANFORD UNIVERSITY

UNIVERSITY OF TOKYO

UNIVERSITY OF UTAH

WASHINGTON STATE UNIVERSITY

UNIVERSITY OF WASHINGTON

AMERICAN MATHEMATICAL SOCIETY NAVAL WEAPONS CENTER 


\section{Pacific Journal of Mathematics}

\section{Vol. 55, No. $1 \quad$ September, 1974}

Robert Lee Anderson, Continuous spectra of a singular symmetric

differential operator on a Hilbert space of vector-valued functions . . . $\quad 1$

Michael James Cambern, The isometries of $L^{p}(X, K) \ldots \ldots \ldots \ldots \ldots . . \ldots$

R. H. Cameron and David Arne Storvick, Two related integrals over spaces of continuous functions ................................

Gary Theodore Chartrand and Albert David Polimeni, Ramsey theory and

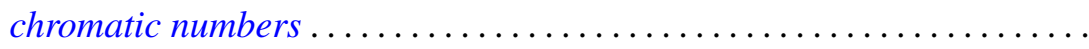

John Deryck De Pree and Harry Scott Klein, Characterization of collectively compact sets of linear operators ...................

John Deryck De Pree and Harry Scott Klein, Semi-groups and collectively compact sets of linear operators ....................... 55

George Epstein and Alfred Horn, Chain based lattices.............. 65

Paul Erdős and Ernst Gabor Straus, On the irrationality of certain series . . 85

Zdeněk Frolík, Measurable uniform spaces................... 93

Stephen Michael Gagola, Jr., Characters fully ramified over a normal

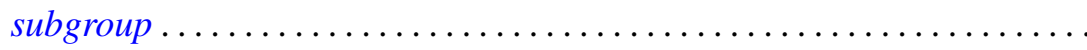

Frank Larkin Gilfeather, Operator valued roots of abelian analytic

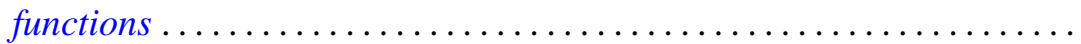

D. S. Goel, A. S. B. Holland, Cyril Nasim and B. N. Sahney, Best approximation by a saturation class of polynomial operators

James Secord Howland, Puiseux series for resonances at an embedded

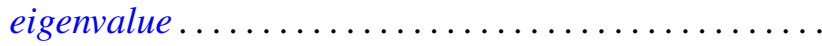

David Jacobson, Linear GCD equations .................

P. H. Karvellas, A note on compact semirings which are multiplicative

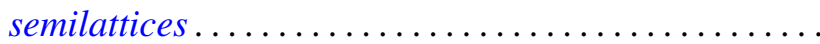

Allan Morton Krall, Stieltjes differential-boundary operators. II . .

D. G. Larman, On the inner aperture and intersections of convex sets

S. N. Mukhopadhyay, On the regularity of the $P^{n}$-integral and its application to summable trigonometric series ....... .

Dwight Webster Read, On $(J, M, m)$-extensions of Boolean algebras ....

David Francis Rearick, Multiplicativity-preserving arithmetic power series.

Indranand Sinha, Characteristic ideals in group algebras

Charles Thomas Tucker, II, Homomorphisms of Riesz spaces . . .

Kunio Yamagata, The exchange property and direct sums of indecomposable injective modules. 